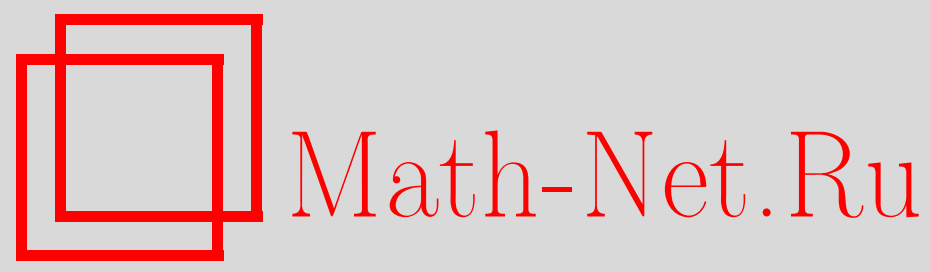

K. А. Волосов, Об одном свойстве анзаца метода Хироты для квазилинейных параболических уравнений, Матем. заметки, 2002, том 71, выпуск 3, 373-389

DOI: https://doi.org/10.4213/mzm353

Использование Общероссийского математического портала Math-Net.Ru подразумевает, что вы прочитали и согласны с пользовательским соглашением http://www . mathnet.ru/rus/agreement

Параметры загрузки:

IP : 3.85 .7 .115

26 апреля 2023 г., 18:17:01

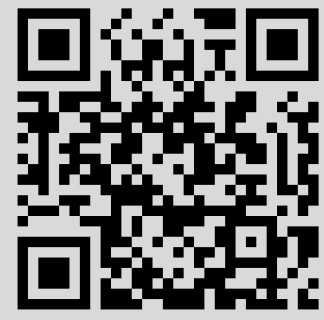




\title{
ОБ ОДНОМ СВОЙСТВЕ АНЗАЦА МЕТОДА ХИРОТЫ ДЛЯ КВАЗИЛИНЕЙНЫХ ПАРАБОЛИЧЕСКИХ УРАВНЕНИЙ
}

\author{
К. А. Волосов
}

В работе устанавливается замкнутость классов дробно рациональных решений ряда нелинейных уравнений, основанная на обнаруженных новых инвариантных свойствах анзаца метода Р. Хироты. Это позволяет строить новые решения для некоторого выделенного класса диссипативных уравнений. Этот алгоритм похож на метод "одевания" решений для интегрируемых уравнений. Полученные уравнения, из которых следует условие "согласования", называются нелинейной парой Лакса с переменньми коэффичиентами. Таким образом, предлагается способ построения таких пар. Для построения решений более сложного вида предлагается использовать экспериментально обнаруженное "свойство нулевых знаменателей и факторизованных скобок". Построенные выражения называем "квази инвариантами"; они позволяют найти правильне соотношения между функциями, входящими в анзац, исправить анзац и построить решение. Приведены примеры новых решений, построенных по предложенной схеме. Такие решения могут быть полезными для мажорирования в теоремах сравнения, при моделировании фазовых процессов и процессов в нейро компьютерах. Написана программа расчета решений методами компьютерной алгебры. Эти приемы дополняют классические методы построения решений, использующие их групповые свойства.

Библиография: 29 названий.

1. Возможность размножения решений уравнений с диссипацией. Опишем сначала простую процедуру построения по паре функций третьей, которая (при определенных условиях на исходную пару) позволяет размножать решения квазилинейных параболических уравнений.

Рассмотрим пару функций $Q(x, t), M(x, t) \in C\left[\mathbb{R}^{1} \times \mathbb{R}_{+}^{1}\right]$ и поставим им в соответствие

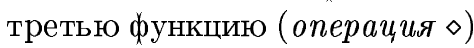

$$
u(x, t)=Q \diamond M=\frac{Q(x, t)+M(x, t) \exp (H(x, t))}{1+\exp (H(x, t))},
$$

где функция $H(x, t)$ имеет вид

$$
H(x, t)=C_{1} \int(M(x, t)-Q(x, t)) d x,
$$

$C_{1}$ - константа.

Справедливы следующие свойства:

(а) коммутативность $M(x, t) \diamond Q(x, t)=Q(x, t) \diamond M(x, t)$;

(b) каждьй элемент в квадрате равен себе $M(x, t) \diamond M(x, t)=M(x, t)$. 
Доказательство пункта (а) следует из смены знака в интеграле (1.2).

Вначале приведены примеры решений, построенные по предлагаемой схеме, которые описьвают взаимодействия “кинков" (решения не ОДУ, а уравнений в частных производных), а затем сформулирован общий результат. Конечно, для того чтобы продемонстрировать решения, описывающие взаимодействие “кинков", и показать, что множество решений непусто и что, используя описанные ниже приемы, можно построить новые решения, коэффициенты в примерах 2-4 должны иметь специальный вид (причина этого объяснена ниже).

При этом их вид вычисляется при применении теоремы 1.3 (см. коментарии в примере 4). В противном случае (пример 5) приходится довольствоваться ОДУ или квадратурой.

ПримеР 1. Рассмотрим известное в теории волн уравнение Фитцхью-Нагумо-Семенова (FHNS):

$$
u_{t}-k_{0} u_{x x}-\varphi_{1} u+\varphi_{3} u^{3}=0,
$$

где $k_{0}=\varphi_{3} /\left(2 a^{2}\right), \varphi_{1}=\varphi_{3}-$ заданные константы [3, с. 64], [4]. Будем искать решение в виде дроби [5]

$$
u(x, t)=\frac{G(x, t)}{F(x, t)} .
$$

Подставим это выражение в уравнение (1.2). После умножения полученного выражения на $F(x, t)^{3}$ для функций $G(x, t)$ и $F(x, t)$ получим однородное уравнение третьего порядка однородности по совокупности этих функций.

Выберем эти функции в виде

$$
G(x, t)=Q(x, t)+M(x, t) \exp (H(x, t)), \quad F(x, t)=1+\exp (H(x, t)) .
$$

Функция $H(x, t)$ имеет вид $(1.2)$, где $C_{1}=\sqrt{\varphi_{3} /\left(2 k_{0}\right)}$.

Процесс построения решений (одевания решений) можно начинать с известных тривиальных решений $u=0, u= \pm 1$.

Выберем решения уравнения (1.3) (ранее это уравнение рассматривалось в [6, с. 17], [3, с. 51], [4], [5], [7]-[12]), для которых выполнена теорема 1.2 (т.е. годятся не все решения, а только те, которые удовлетворяют условию согласования, см. ниже) и параллельно объясним закономерность перехода констант сдвига в новые решения.

Рассмотрим функции $u(x, t)=M(x, t)$ или $u(x, t)=Q(x, t)$; это простейшие решения:

$$
\begin{gathered}
M(x, t)=-\left(1+\exp \left(m_{0}+a x+b t\right)\right)^{-1}, \\
Q(x, t)=\frac{-1+\exp \left(2 a x+q_{0}\right)}{1+\exp \left(2 a x+q_{0}\right)}, \quad b=-\frac{3 \varphi_{3}}{2},
\end{gathered}
$$

где $m_{0}, q_{0}$ - заданные константы. Убедимся в том, что для них вьполнено условие согласования (см. теорему 1.2), и вычислим функцию (1.2):

$$
H(x, t)=\ln \frac{1+\exp \left(m_{0}+a x-3 t \varphi_{3} / 2\right)}{1+\exp \left(q_{0}+2 a x\right)} ;
$$

получим следующее решение (1.1) (кинк, см. рис. 1):

$$
u_{1}=\frac{1-\exp \left(-q_{0}-2 a x+\ln 2\right)}{1+\exp \left(-q_{0}-2 a x+\ln 2\right)+\exp \left(m_{0}-q_{0}-a x-3 t \varphi_{3} / 2\right)} .
$$


Этот факт проверяется прямой подстановкой в уравнение (1.3). Если функции $M, Q$ не удовлетворяют условию согласования, то функция $u_{1}$ не является решением. Исходные константы сдвига $q_{0}, m_{0}$ перешли в новое решение и к ним добавилась константа $\ln 2$.

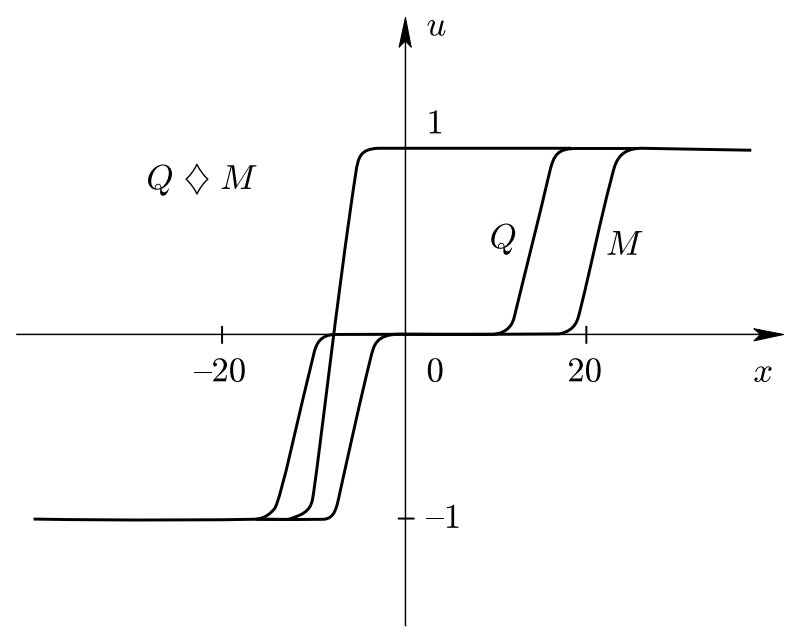

Рис. 1

Существует следующий вариант функций, для которых также вьполнены условия теоремы 1.2:

$$
\begin{aligned}
M(x, t) & =\left(1+\exp \left(m_{1}-a x+b t\right)\right)^{-1}, \\
Q(x, t) & =\frac{1-\exp \left(q_{1}-2 a x\right)}{1+\exp \left(q_{1}-2 a x\right)}, \quad b=-\frac{3 \varphi_{3}}{2},
\end{aligned}
$$

где $m_{1}, q_{1}$ - заданные константы.

Функция $H(x, t)(1.2)$ имеет вид

$$
H(x, t)=\ln \frac{1+\exp \left(m_{1}-a x-3 t \varphi_{3} / 2\right)}{1+\exp \left(q_{1}-2 a x\right)} ;
$$

получим следующее (второй кинк) решение:

$$
u_{2}=-\frac{1-\exp \left(-q_{1}+2 a x+\ln 2\right)}{1+\exp \left(-q_{1}+2 a x+\ln 2\right)+\exp \left(m_{1}-q_{1}+a x-3 t \varphi_{3} / 2\right)} .
$$

Выберем в качестве новых функции $Q(x, t)=u_{1}, M(x, t)=u_{2}$ - решения уравнения (1.3) и вычислим из (1.2) $H(x, t)$ и следующее решение:

$$
\begin{array}{r}
H(x, t)=\ln \frac{1+2 \exp \left(-q_{1}+2 a x\right)+\exp \left(m_{1}-q_{1}+a x-3 t \varphi_{3} / 2\right)}{2+\exp \left(q_{0}+2 a x\right)+\exp \left(m_{0}+a x-3 t \varphi_{3} / 2\right)}, \\
u_{3}=\frac{-1+\frac{1}{3} \exp \left(q_{0}+2 a x\right)+\frac{2}{3} \exp \left(-q_{1}+2 a x\right)}{1+\frac{1}{3} \exp \left(q_{0}+2 a x\right)+\frac{2}{3} \exp \left(-q_{1}+2 a x\right)+\frac{1}{3} \exp \left(m_{0}+a x-3 t \varphi_{3} / 2\right)} . \\
+\frac{1}{3} \exp \left(m_{1}-q_{1}+a x-3 t \varphi_{3} / 2\right)
\end{array} .
$$


Из этой формулы можно понять, как сконструирована константа сдвига (т.е. как происходит взаимодействие). Действительно, в переменных $\tau_{1}=-a x+3 t \varphi_{3} / 2, \tau_{2}=a x+$ $3 t \varphi_{3} / 2$ двухфазное решение (1.3) имеет вид

$$
\begin{gathered}
u_{3}\left(\tau_{1}, \tau_{2}\right)=\frac{-c_{1} \exp \left(\tau_{1}\right)+c_{2} \exp \left(\tau_{2}\right)}{1+c_{1} \exp \left(\tau_{1}\right)+c_{2} \exp \left(\tau_{2}\right)}, \\
c_{1}=\frac{\exp \left(q_{1}\right)\left(2+\exp \left(q_{0}\right)\right)}{\exp \left(q_{0}+m_{1}\right)+\exp \left(q_{1}+m_{0}\right)}, \quad c_{2}=\frac{\exp \left(q_{0}\right)\left(2+\exp \left(q_{1}\right)\right)}{\exp \left(q_{0}+m_{1}\right)+\exp \left(q_{1}+m_{0}\right)} .
\end{gathered}
$$

Такие решения изучались ранее $[13$, с. 190] и в [3, с. 51], [4], [5], [10]-[12], [14], [15].

Подведем итоги примера. "Стартуем" с четырех решений $(1.6),(1.9)$. По ним строим решения (1.8), (1.11). Для решений (1.8), (1.11) выполнено условие согласования, приведенное ниже в теореме 1.1. Далее, из описанного множества решений можно выбрать любую пару (она автоматически будет удовлетворять условию согласования) и построить новое решение. По-видимому, механизм взаимодействия решений (1.1) и переход констант сдвига (см. рис. 1) являются новымы. Новые решения для уравнения (1.3) построены ниже.

ПРимеР 2. Рассмотрим модифицированное уравнение Аллена-Кана. Это уравнение связано с задачей о фазовом переходе (см., например, [11]). Это решение построено по схеме, изложенной в п. 2.

Уравнение имеет вид

$$
\varepsilon\left(a_{1}-h_{1} u\right) u_{t}-\varepsilon^{2}\left(a_{1}-h_{1} u\right) u_{x x}-\varepsilon h_{1}\left(a_{1}+h_{1} u\right) u_{x}-\varepsilon^{2} 2 h_{1}\left(u_{x}\right)^{2}-\frac{1}{2} a_{1}\left(a_{1}^{2}+h_{1}^{2}\right) u\left(1-u^{2}\right)=0,
$$

где $\varepsilon<1$ - мальй пераметр, а $a_{1}, h_{1}$ - заданные константы. (Вид коэффициентов вычислен при применении теоремы 1.3.)

Новое решение, построенное по схеме, приведенной в п. 2 , имеет вид

$$
u(x, t)=\frac{1-\exp \left(a_{1} x / \varepsilon\right)}{1+\exp \left(a_{1} x / \varepsilon\right)+\exp \left(\left(\left(-h_{1}^{2}-3 a_{1}^{2}\right) t+2\left(h_{1}+a_{1}\right) x\right) /(4 \varepsilon)\right)}
$$

и описывает развитие второй волны из малого возмущения в присутствии другой волны (см. рис. 2). Это взаимодействие "кинков", распространяющихся в полосе $u \in[-1,1], u \in[0,1]$. Существует большой цикл работ (см., например, библиографию в [11], [16]-[19]), в которых рассматривается система двух уравнений. А именно, если уравнение типа (1.14) дополняется уравнением теплопроводности, то эта система описывает модифицированную задачу Стефана. Построенное решение (1.15) в пределе (при стремлении малого параметра к нулю) содержит две тета-функции Хевисайда и может моделировать три состояния $(-1,0,1)$ : жидкое, промежуточную фазу и твердое состояние. Хочется надеяться, что построенное решение будет полезным в таких задачах.

ПРИмеР3. Рассмотрим уравнение диффузии примеси хлора в кремнии. Этот процесс рассматривался нами в [13, с. 126] и [20]. Там доказано, что решение в виде самоподобной двигающейся структуры существует. Сушествуют решения, у которых скорость переднего и заднего фронта волны различные и структура "размазывается". Здесь приведем точное решение (синергета диссипативной структуры [13]) по схеме, изложенной в п. 2. 


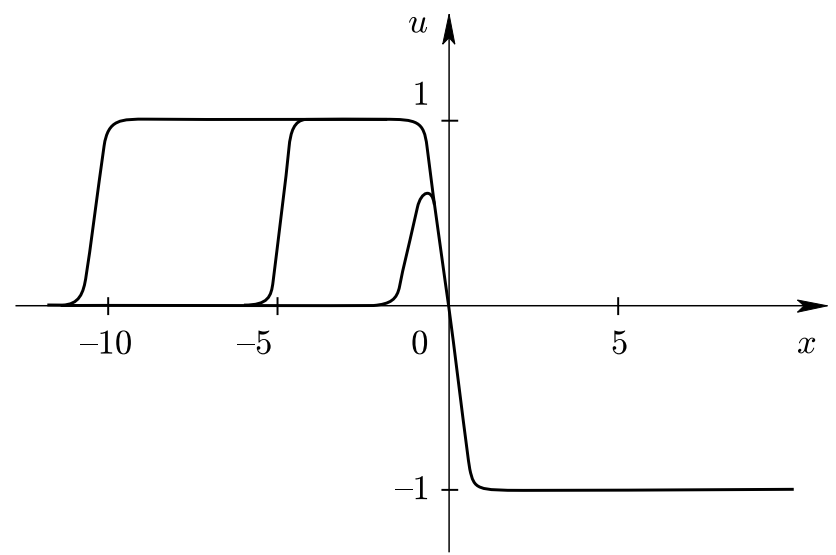

Рис. 2

Уравнение имеет вид

$$
\left(b_{1} u+b_{2} u^{2}\right) u_{t}-\left(k_{0}+k_{1} u\right) u_{x x}-g_{0}\left(u_{x}\right)^{2}-\left(a_{1}-u\right)\left(a_{2}-u\right) u(-1+u)=0,
$$

где $a_{1}, a_{2}, b_{1}, b_{2}, k_{0}, k_{1}, g_{0}$ - заданные константы.

Функция $\left(a_{1}-u\right)\left(a_{2}-u\right) u(-1+u)$ в работах [13], [3] называется функиией сто-

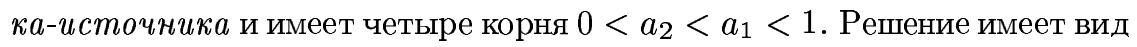

$$
u[x, t]=\frac{\exp \left(-a_{1} t / b_{2}+a x\right)\left(1+a_{1} C \exp \left(-a_{1} t / b_{2}+a x\right)\right)}{1+2 \exp \left(-a_{1} t / b_{2}+a x\right)+C \exp \left(2\left(-a_{1} t / b_{2}+a x\right)\right)} .
$$

Решение описьвает взаимодействие волн, распространяющихся в полосах $u \in[0,1]$ и $u \in\left[a_{1}, 1\right]$ (см. рис. 3 ). Константы в решении равны

$$
\begin{aligned}
& b_{1}=-b_{2} \frac{-8+12 a_{1}-5 C+4 a_{1} C+3 C^{2}}{2(C-1)^{2}} \\
& k_{0}=-a_{1} \frac{-4+4 a_{1}+C}{2 a^{2}(C-1)^{2}} \\
& g_{0}=\frac{7-11 a_{1}+4 a_{1}^{2}-2 C-2 a_{1} C+3 a_{1}^{2} C+a_{1} C^{2}}{a^{2}(C-1)^{2}} \\
& k_{1}=-\frac{8-12 a_{1}+4 a_{1}^{2}-2 C-3 a_{1} C+4 a_{1}^{2} C+a_{1} C^{2}}{2 a^{2}(C-1)^{2}} \\
& a_{2}=\frac{1}{3(C-1)^{2}}, \quad a_{1}=\frac{10-3 C}{12}
\end{aligned}
$$

ПРИмЕР 4. У давно известного уравнения

$$
u_{t}-k_{0} u_{x x}-\left(h_{1}+h_{2} u\right) u_{x}-s_{1} u\left(-s_{2}+u\right)\left(s_{2}+u\right)=0
$$

существуют свойства, которые, как это ни странно, не обсуждались ранеев литературе. Тест Пенлеве [14] дает следующий результат. 


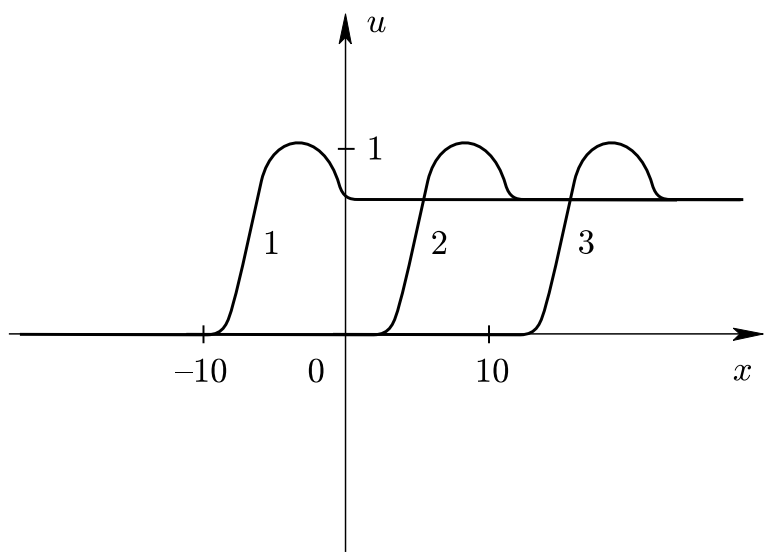

Рис. 3

УтвеРЖДЕнИЕ 1.1. Решение уравнения (1.19) в виде ряда Лорана по переменной $а x+b t$ имеет полюс первого порядка, а дисперсионное уравнение имеет вид $\left(b+a h_{1}\right) A\left(b, a, k_{0}, h_{1}, h_{2}, s_{1}, s_{2}\right)=0$, причем решение довольно громоздкого алгебраического уравнения $A\left(b, a, k_{0}, h_{1}, h_{2}, s_{1}, s_{2}\right)=0$ относительно $b$ может иметь несколько вещественных корней.

Это означает, что существует как миниум два семейства решений, соответствующих волнам, распространяюшимися с двумя различными скоростями. Приведем пример в упрощенном случае.

УТВЕРЖДЕНИЕ 1.2. Множество точныХ решений уравнения

$$
u_{t}-k_{0} u_{x x}-\frac{3 a_{1} k_{0} u u_{x}}{s_{2}}-\frac{a_{1}^{2} k_{0} u\left(-s_{2}+u\right)\left(s_{2}+u\right)}{s_{2}^{2}}=0
$$

где $h_{1}=0, h_{2}=3 a_{1} k_{0} / s_{2}, s_{1}=a_{1}^{2} k 0 / s_{2}^{2}, a k_{0}, a_{1}, s_{2}$ - заданные константы, строится по теореме 1.2 путем композичии решений двух подмножеств. Первое подмножество представлено функииями - решениями (1.20), которые зависят только от $x$, и имеет вид

$$
\begin{gathered}
M=\frac{s_{2}\left(-1+\exp \left(2 a_{1} x\right)\right)}{1+\sqrt{2} \exp \left(a_{1} x\right)+\exp \left(2 a_{1} x\right)}, \quad Q=\frac{s_{2}}{1+\exp \left(-a_{1} x\right)} \\
M_{0}=\frac{s_{2}\left(-1+\exp \left(2 a_{1} x\right)+\exp \left(a_{1}(c+2 x)\right)+\exp \left(a_{1}\left(c_{1}+2 x\right)\right)\right)}{1+(2+\sqrt{2}) \exp \left(a_{1} x\right)+\exp \left(2 a_{1} x\right)+\exp \left(a_{1}(c+2 x)\right)+\exp \left(a_{1}\left(c_{1}+2 x\right)\right)} \\
Q_{0}=\frac{s_{2}\left(\exp \left(a_{1}(c+x)\right)+\exp \left(a_{1}\left(c_{1}+x\right)\right)\right)}{2+\exp \left(a_{1}(c+x)\right)+\exp \left(a_{1}\left(c_{1}+x\right)\right)},
\end{gathered}
$$

где функиия $H(x, t)$ в формуле (1.1)

$$
H(x, t)=-\frac{a_{1}}{s_{2}} \int(M(x, t)-Q(x, t)) d x ;
$$


второе подмножество волн (кинки) представлено решениями

$$
\begin{aligned}
M(x, t) & =\frac{s_{2}\left(-1+\exp \left(a_{1} x\right)\right)}{1+\exp \left(a_{1} x\right)+\exp \left(3 k_{0} a_{1}^{2} t / 4+a_{1} x / 2\right)}, \\
Q(x, t) & =\frac{s_{2}\left(-2+\exp \left(a_{1} x\right)\right)}{2+\exp \left(a_{1} x\right)+2 \exp \left(3 k_{0} a_{1}^{2} t / 4+a_{1} x / 2\right)} \\
H(x, t) & =b t-\frac{a_{1}}{2 s_{2}} \int(M(x, t)-Q(x, t)) d x
\end{aligned}
$$

где $C_{0}, c_{1}, c, n$ - произвольные константы, $b$ - неизвестная константа.

ДоКАЗАТЕЛЬСТво. Свойства Этого уравнения отличаются от остальных. Это отличие состоит в двух пунктах:

a) решения каждого из подмножеств независимо размножаются; константа $b$ определяется после подстановки окончательного анзаца в уравнение (1.20);

b) в одно решение могут входить разные константы сдвига (1.22).

ЗАмЕчАнИЕ 1.1. Построенные решения могут дополнить обширные справочные материалы [21], так как с их помощью можно вычислить новые решения уравнения Абеля второго рода в параметрической форме.

Приведем основной результат. Все доказательства могут быть проведены вручную в простейших случаях, если положить все коэффициенты равными единище. В общем случае вычисления производились в символьном виде.

Рассмотрим квазилинейное параболическое уравнение

$$
\left(1+b_{1} u+b_{2} u^{2}\right) u_{t}-\left(h_{1}+h_{2} u+h_{3} u^{2}\right) u_{x}-g_{0}\left(u_{x}\right)^{2}-\left(k_{0}+k_{1} u\right) u_{x x}+\sum_{i=1}^{4} \varphi_{i} u^{i}=0
$$

где $b_{j}, h_{j}, k_{j-1}(j=1,2), \varphi_{i}(i=1,2,3,4), g_{0}$ - заданные константы.

Предполагается, что коэффициенты при $u_{t}$ и $u_{x x}$ не обращаются в нуль в области изменения функции $u$.

Будем искать решение в виде дроби (1.4). Подставим ее в уравнение (1.25). После умножения полученного выражения на $F(x, t)^{4}$ для функций $G(x, t)$ и $F(x, t)$ получим однородное уравнение четвертого порядка однородности по совокупности этих функций.

Частньй случай уравнения (1.25) имеет вид

$$
\left(1+b_{1} u\right) u_{t}-\left(h_{1}+h_{2} u\right) u_{x}-k_{0} u_{x x}+\sum_{i=1}^{3} \varphi_{i} u^{i}=0
$$

Если сделать замену (1.4), то после умножения полученного выражения на $F(x, t)^{3}$ оно приводится к уравнению третьего порядка однородности для функций $G(x, t)$ и $F(x, t)$.

Частные случаи уравнения (1.25) широко известны (см. обширную библиографию в [3], [6], [12]-[14], [22]-[25]).

Существенным отличием данной работы от всех предыдущих (идею и предварительные данные можно найти в $[1],[2],[26])$ является обнаружение нового свойства решений данного класса уравнений (сравни с [27], [28]). 
Рассматривая различные формы записи решения по методу Хироты, можнн прийти к заключению, что все они приводят $\kappa$ виду (1.5), остается только одна возможсность расиирения этой записи, а именно: добавление отрезков ряда по степеням экспонент $\exp (i H(x, t)), i=2, \ldots$.

Подставим (1.5) в (1.4), а затем в (1.26) и приравняем к нулю коэффициенты при одинаковых степенях $\exp (H(x, t))$ (эти степени суть $0,1,2,3)$. Получим систему уравнений, которую обозначим (eq 0), (eq 1), (eq 2), (eq 3 ).

Уравнение (еq 0$)$ является тем же исходньм уравнением $(1.26)$ для функции $Q(x, t)$. Следовательно, функция $Q(x, t)$ - решение этого уравнения.

В данной работе решения вида (1.1) по степени сложности (количеству слагаемых с множителем степени $\exp (i H))$ назьваются решениями первой, второй и т.д. моды.

ОпРЕДЕЛЕниЕ 1.1. Будем назьвать запись решения уравнения (1.26) вида

$$
u(x, t)=\frac{\exp (H(x, t)) M(x, t)+Q(x, t)}{1+\exp (H(x, t))},
$$

где $M(x, t), Q(x, t)$ - некоторые решения уравнений (1.26), формой записи решений первой моды, запись решения в виде

$$
u(x, t)=\frac{\exp (H(x, t)) M(x, t)+\exp (2 H(x, t)) R(x, t)+Q(x, t)}{1+Z_{1}(x, t) \exp (H(x, t))+\exp (2 H(x, t)) Z_{2}(x, t)}
$$

формой записи решений второй моды, и т.д.

Справедлива

ТЕОРема 1.1. Пусть функция $M$ является решением уравнения

$$
\left(1+b_{1} M\right) M_{t}-\left(h_{1}+h_{2} M\right) M_{x}-k_{0} M_{x x}+\sum_{i=1}^{3} \varphi_{i} M^{i}=0,
$$

а функиия $Q$ является другим решением (того жее) уравнения

$$
\left(1+b_{1} Q\right) Q_{t}-\left(h_{1}+h_{2} Q\right) Q_{x}-k_{0} Q_{x x}+\sum_{i=1}^{3} \varphi_{i} Q^{i}=0,
$$

и эти же функиии $M$ и $Q$ дополнительно связаны между собой соотношением согласования

$$
\begin{aligned}
(M- & Q) b_{1} k_{0} H_{x x}(x, t)+H_{x}(x, t)\left(M\left(b_{1} h_{1}-h_{2}\right)+Q\left(-b_{1} h_{1}+h_{2}\right)+2 b_{1} k_{0}\left(M_{x}-Q_{x}\right)\right) \\
& +\left(2+M b_{1}+Q b_{1}\right) k_{0} H_{x}(x, t)^{2}+(M-Q)^{2}\left(b_{1} \varphi_{2}+\left(-1+b_{1}(M+Q)\right) \varphi_{3}\right) \\
& +b_{1}^{2}(M-Q)\left(M_{t}-Q_{t}\right)-b_{1} h_{2}(M-Q)\left(M_{x}-Q_{x}\right)=0
\end{aligned}
$$

а функиия $H(x, t)$ удовлетворяет уравнению

$$
-(M-Q) b_{1} Q^{2} H_{t}(x, t)+H_{x}(x, t) Q^{2}\left(M h_{2}-Q h_{2}-2 k_{0} H_{x}(x, t)\right)+M(M-2 Q)=0 .
$$

Тогда функиия $u(x, t)$ вида (1.27) является решением уравнения (1.26). 
ЗАмечаниЕ 1.2. Уравнение (1.31) есть уравнение Рикатти для функции $H_{x}(x, t)$, а уравнение (1.32) является уравнением Гамильтона-Якоби для функции $H(x, t)$.

Уравнения (1.31), (1.32) вызывают ассоциацию с парами Лакса с переменными коэффициентами, см. ниже.

ЗАмЕчаниЕ 1.3. Уравнение (1.32) может иметь другой вид записи [26]

$$
-(M-Q) b_{1} H_{t}(x, t)+H_{x}(x, t)\left(M h_{2}-Q h_{2}-2 k_{0} H_{x}(x, t)\right)+(M-Q)^{2} \varphi_{3}=0 .
$$

Положим $b_{1}=0$; в этом случае имеются явные формулы для $H(x, t)$. Из теоремы 1.1 немедленно получаем следствие.

ТЕОРема 1.2. Пусть $b_{1}=0$, функиия $M$ является решением уравнения

$$
M_{t}-\left(h_{1}+h_{2} M\right) M_{x}-k_{0} M_{x x}+\sum_{i=1}^{3} \varphi_{i} M^{i}=0,
$$

функиия $Q$ является решением уравнения

$$
Q_{t}-\left(h_{1}+h_{2} Q\right) Q_{x}-k_{0} Q_{x x}+\sum_{i=1}^{3} \varphi_{i} Q^{i}=0
$$

и эти жсе функиии $M$ и $Q$ дополнительно связаны между собой соотношением согласования

$$
\begin{aligned}
& -\left(\int\left(M_{t}-Q_{t}\right) d x\right) 2\left(h_{2} \pm m\right)+\left(M^{2}-Q^{2}\right)\left(h_{2}^{2}+12 k_{0} \varphi_{3} \pm h_{2} m\right) \\
& \quad+2(M-Q)\left(h_{1} h_{2}+4 k_{0} \varphi_{2} \pm h_{1} m\right) \\
& \quad+8 k_{0} C 0^{\prime}(t)+2 k_{0}\left(-h_{2} \pm 3 m\right)\left(M_{x}-Q_{x}\right)=0
\end{aligned}
$$

әде $m=\sqrt{h_{2}^{2}+8 k_{0} \varphi_{3}}$, а функиия $H(x, t)$ имеет вид

$$
H(x, t)=C 0(t)+\frac{h_{2} \pm \sqrt{h_{2}^{2}+8 k_{0} \varphi_{3}}}{4 k_{0}} \int(M(x, t)-Q(x, t)) d x
$$

Тогда функиия $u(x, t)$ вида (1.27) является решением уравнения (1.26).

СХемА ДОКАЗАТЕЛЬСТВА. Приведем краткую схему доказательства теорем. Подставим (1.27) в (1.26), приведем к общему знаменателю и рассмотрим числитель полученного уравнения.

Приравняем к нулю коэффициент при экспоненте в нулевой степени. Полученное уравнение снова является в точности уравнением $(1.26)$ для функции $Q(x, t)$. Следовательно, функция $Q(x, t)$ - решение этого уравнения.

Уравнение, которое возникает после приравнивая к нулю коэффициента при экспоненте $\exp (3 H(x, t))$, будем назьвать уравнением (eq 3$)$. Это уравнение является в точности уравнением (1.26) для функции $M(x, t)$. Следовательно, функция $M(x, t)$ - решение этого уравнения. 
Уравнение, которое возникает после приравнивая к нулю коэффициента при экспоненте $\exp (H(x, t))$, будем называть (еq 1$)$.

Уравнение, которое возникает после приравнивая к нулю коэффициента при экспоненте $\exp (2 H(x, t))$, будем назьвать (еq 2).

Пользуясь тем, что функции $M$ и $Q$ удовлетворяют уравнению (1.26), исключим из уравнений (eq 1) и (eq 2) вторые производные $M_{x x}(x, t)$ и $Q_{x x}(x, t)$.

Из уравнения (eq 1$)$ можно выразить $H_{x x}(x, t)$ и подставить в уравнение (eq 2$)$. Тогда получим уравнение Гамильтона-Якоби (1.32). В условиях теоремы $1.2\left(\right.$ при $\left.b_{1}=0\right)$ из него естественно определяется функция $H(x, t)(1.37)$. Выразим из уравнения (1.32) производную $H_{t}(x, t)$ и подставим в уравнение $($ еq 1$)$; получим условие согласования $(1.31)$ или (1.36) соответственно. Если подставить производную $H_{t}(x, t)$ в уравнение (eq 2$)$, то также получим условие согласования (т.е. два уравнения совпадают). В заключение отметим, что существуют два варианта соотношений, приведенных в теореме; они отличаются знаками.

Из приведенных теорем фактически следует процедура построения новых решений уравнений (аналог суперпозиции решений), которая связана со свойствами уравнения (1.26) и рассматриваемого анзаца. С помощью этой процедуры построены все решения в приведенных примерах.

Интересно отметить, что условие согласования (1.36) после дифференцирования по $x$ можно рассматривать как модифицированное уравнение Бюргерса с потенциалом относительно (симметрично) одной из функций $M(x, t)$ или $Q(x, t)$.

В следующей теореме описьвается одно интересное свойство: если есть две пары решений, которые удовлетворяют условиям согласования, то по ним строится последовательность решений, которые попарно удовлетворяют условию согласования.

ТеОрема 1.3. Пусть $b_{1}=0$ в уравнении (1.26), две пары функиий $M_{0}, Q_{0} u$ $M_{1}, Q_{1}$ являются решениями уравнений

$$
\begin{gathered}
M_{j_{t}}-\left(h_{1}+h_{2} M_{j}\right) M_{j_{x}}-k_{0} M_{j_{x x}}+\sum_{i=1}^{3} \varphi_{i} M_{j}^{i}=0 \\
Q_{j_{t}}-\left(h_{1}+h_{2} Q_{j}\right) Q_{j_{x}}-k_{0} Q_{j_{x x}}+\sum_{i=1}^{3} \varphi_{i} Q_{j}^{i}=0
\end{gathered}
$$

где $j=0,1$, для кажсдй пары выполнены условия согласования

$$
\begin{gathered}
\left(\int\left(M_{j_{t}}-Q_{j_{t}}\right) d x\right) 4\left(h_{2}-m\right)-2\left(M_{j}^{2}-Q_{j}^{2}\right)\left(h_{2}^{2}+12 k_{0} \varphi_{3}-h_{2} m\right) \\
-4\left(M_{j}-Q_{j}\right)\left(h_{1} h_{2}+4 k_{0} \varphi_{2}-h_{1} m\right)+4 k_{0}\left(h_{2}+3 m\right)\left(M_{j_{x}}-Q_{j_{x}}\right)=0 \\
H_{j}(x, t)=-\frac{-h_{2} \pm \sqrt{h_{2}^{2}+8 k_{0} \varphi_{3}}}{4 k_{0}} \int\left(M_{j}(x, t)-Q_{j}(x, t)\right) d x
\end{gathered}
$$

əде $m=( \pm) \sqrt{h_{2}^{2}+8 k_{0} \varphi_{3}}$, две функиии

$$
\begin{aligned}
& Q(x, t)=\frac{Q_{1}(x, t)+M_{1}(x, t) \exp \left(H_{1}(x, t)\right)}{1+\exp \left(H_{1}(x, t)\right)} \\
& M(x, t)=\frac{Q_{0}(x, t)+M_{0}(x, t) \exp \left(H_{0}(x, t)\right)}{1+\exp \left(H_{0}(x, t)\right)}
\end{aligned}
$$


являются решениями уравнения (1.26), и для этих жее функиии $M$ и выполнено соотношение согласования

$$
\begin{aligned}
& \left(\int\left(M_{t}-Q_{t}\right) d x\right) 4\left(h_{2}-m\right)-2\left(M^{2}-Q^{2}\right)\left(h_{2}^{2}+12 k_{0} \varphi_{3}-h_{2} m\right) \\
& \quad-4(M-Q)\left(h_{1} h_{2}+4 k_{0} \varphi_{2}-h_{1} m\right)+4 k_{0}\left(h_{2}+3 m\right)\left(M_{x}-Q_{x}\right)=0
\end{aligned}
$$

әде $m=( \pm) \sqrt{h_{2}^{2}+8 k_{0} \varphi_{3}}$, а функиия $H(x, t)$ имеет вид

$$
H(x, t)=-\frac{-h_{2} \pm \sqrt{h_{2}^{2}+8 k_{0} \varphi_{3}}}{4 k_{0}} \int(M(x, t)-Q(x, t)) d x
$$

Тогда функиия $u(x, t)$ вида

$$
u(x, t)=\frac{Q(x, t)+M(x, t) \exp (H(x, t))}{1+\exp (H(x, t))}
$$

является решением уравнения (1.26) и для пар функиий $\left(u, M_{0}\right),\left(u, Q_{1}\right),\left(M, Q_{0}\right)$, $\left(Q, Q_{1}\right)$ и т.д. условие согласования выполняется без дополнительных условий.

ДокАЗАТЕЛЬСтво проводится прямой подстановкой. Такое же утверждение имеет место и для случая $b_{1}$, отличного от нуля. В этом случае для функций $H_{j}$ справедливы выражения, аналогичные (1.44).

Аналогичные результаты имеют место для уравнения (1.25) с некоторьми ограничениями на коэффициенты. Пусть

$$
\varphi_{1}=a_{2} a_{1}, \quad \varphi_{2}=-\left(a_{1}+a_{2}+a_{1} a_{2}\right), \quad \varphi_{3}=1+a_{1}+a_{2}, \quad \varphi_{4}=-1
$$

Тогда функция стока-источника имеет вид $u\left(a_{1}-u\right)\left(a_{2}-u\right)(1-u)$ (явно выделены четыре корня).

Справедлива следующая теорема.

ТЕоремА 1.4. Пусть решение (1.1) имеет вид (1.27) и реализуется одна из следующих трех ситуаций:

1) $(Q(x, t)$ или $M(x, t))=-k_{0} / k_{1},\left(k_{0}+k_{1}\right)\left(k_{0}+a_{1} k_{1}\right)\left(k_{0}+2 a_{1} k_{1}\right)=0$;

2) $b_{1}=k_{1} / k_{0}, b_{2}=0$;

3) $b_{1}=0, b_{2}=0, k_{1}=0$.

Тогда функиии $M$ и $Q$ также являются решениями уравнения, совпадающего $c$ (1.25), $и$ эти же функиии дополнительно связаны между собой соотношениями согласования (уравнение Риккати для $H_{x}(x, t)$, аналог условия (1.31)) и дополнительно удовлетворяется уравнение Гамильтона-Якоби (аналог условия (1.32)) (әти уравнения не приводим из-за их громоздкости). 
ДокАЗАТЕЛЬСТво аналогично доказательству предыдущих теорем.

Сформулируем предлагаемьй способ построения точных решений (алгоритм программы) уравнения в форме записи анзаца первой моды (1.1) для уравнения (1.26). Возьмем два любых решения $M$ и $Q$ уравнения (1.26). Подставим их в анзац (1.27) с произвольной функцией $H$. Подставим этот анзац в уравнение. Приравняем к нулю коэффициенты при одинаковых степенях экспонент функции $H$. Получим четыре уравнения. Первое и последнее уравнения удовлетворяются тождественно, так как $M$ и $Q$ - решения. Итак, имеем переопределенную систему из двух уравнений на одну функцию $H$ в случае (1.26).

Выражаем вторую производную функции $H_{x \boldsymbol{x}}$ из оставшегося уравнения и подставляем в следующее уравнение. Факторизуем полученные уравнения (см. теоремы 1.1-1.4). Обычно возникает несколько случаев (имеется несколько множителей). Рассматриваем их последовательно, т.е. приравниваем их к нулю. Интегрируя полученные уравнения первого порядка, находим функцию $H$ (предполагаемое выражение). Подставляем это выражение в анзац (1.27). Так как функции $M$ и $Q$ были определены ранее, получим некоторую функцию $u$, которая, возможно, является решением, если выполнено условие согласования. Поэтому для проверки эту функцию еще раз подставляем в уравнение (1.26). Вьпгрьш в вычислениях заключается в том, что не надо стремиться удовлетворить двум оставшимся уравнениям специально. Надо только сделать проверку. Если уравнение (1.26) удовлетворяется тождественно, то построено решение. Иногда найденная функиия является решением только при некоторых ограничениях на коэффициенты уравнения. Это и объясняет конкретньй вид коэффициентов в уравнениях в примерах. Если уравнение не удовлетворяется тождественно, то все проделанные вычисления были напрасны и следует перейти к следующему варианту.

Теперь интерпретируем уравнения (1.31), (1.32) как нелинейную пару соотношений

$$
\begin{aligned}
H_{x x} & =f_{1} H_{x}^{2}+f_{2} H_{x}+f_{3}, \\
H_{t} & =f_{4} H_{x}^{2}+f_{5} H_{x}+f_{6},
\end{aligned}
$$

где коэффищиенты $f_{i}$ - функции от $M(x, t), Q(x, t)$ и их производных. Затем вычисляем $H_{x x t}, H_{t x x}$ и приравниваем их, исключая, как и ранее, $M_{x x}, Q_{x x}, H_{t}, H_{x x}$, получим, что данное соотношение удовлетворяется не тождественно, а на некотором классе важных в приложении уравнений и систем. Затем проверяем выполнение равенства $H_{x t}=H_{t x}$.

Наиболее естественной модификацией метода Хироты является изменение формы анзаца решения (1.1) на вид

$$
u(x, t)=Q \diamond M=\frac{F(Q, Z)+G(M, Z) Z(x, t)}{1+Z(x, t)},
$$

где $M, Q$ - решения исходного уравнения, а $F, G, Z$ - неизвестные функции. Подставим выражение $(1.47)$ в (1.25) и, исключая, как и ранее, $M_{x x}, Q_{x x}$, получим некоторое выражение. Разбивая это выражение на две части различными способами по аналогии c (1.46), получаем нелинейные пары соотношений. Далее, изучаем, при каких условиях выполнено равенство смешанных производных

$$
\frac{\partial}{\partial t} Z_{x x}=\frac{\partial^{2}}{\partial x^{2}} Z_{t}, \quad z_{x t}=z_{t x}
$$

Получаем соотношения параметров и коэффициентов уравнения и выбираем вид функций $F, G$. 
2. Свойство “нулевых знаменателей и факторизованных скобок". Далее опишем "экспериментально" обнаруженное свойство "нулевых знаменателей и факторизованных скобок" системы уравнений, которая возникает при применении метода Хироты. Для этого продолжим рассмотрение примера 1.

ПРимеР 1 (продолжение). Рассмотрим анзац решения второй моды

$$
u(x, t)=\frac{\exp (H(x, t)) Q_{1}(x, t)+\exp (2 H(x, t)) Q_{2}(x, t)-Q(x, t)}{1+Z_{1}(x, t) \exp (H(x, t))+\exp (2 H(x, t)) Z_{2}(x, t)}
$$

Подставим это выражение в уравнение (1.3) и получим переопределенную систему семи уравнений на четыре функции. Организуем следующую процедуру вычислений. Из произвольного уравнения выразим сначала одну из старших производных любой функции. Если при этом получится дробь, знаменатель этой дроби запишем в таблицу. Подставим выраженную производную во все остальные уравнения. Применим ко всем следующим уравнениям процедуру факторизации (т.е. выделение общего множителя). Это стандартная процедура в системах символьных вычислений. Возникающие общие множители также заносим в таблищу. Из произвольного уравнения вновь полученной системы выразим одну из старших производных любой другой функции (если их нет, то первую производную). Если при этом получится дробь, знаменатель этой дроби запишем в таблицу. Подставим выраженную производную во все остальные уравнения. Применим ко всем следующим уравнениям процедуру факторизации (т.е. выделение общего множителя). Повторяя такие операции, получим в таблице список выражений, содержащих функции (а возможно, и их производные) из анзаца (2.1). Будем называть выражения в таблице квазиинвариантами. Будем теперь приравнивать к нулю некоторое (произвольное) количество этих выражений. Если из полученных равенств удается найти выражения одних функций через другие, подставим их в анзац (2.1). Оказьвается (по непонятньм пока причинам), эти действия позволяют найти "правильные" соотношения меж ду функциями, и в результате, как правило, получаются решения (возможно, тривиальные и известные, а иногда и новые). Если из наших равенств (квазиинвариантов) удалось выразить часть функций через другие, мы подставляем эти выражения в (2.1), получаем новьй анзац (которьй содержит меньшее число неизвестных функций) и повторяем процедуру. Таким способом были получены решения, приведенные в работе. Нам неясно, какие математические утверждения лежат в основе этих экспериментально обнаруженных явлениях, но похоже, что они связаны с "симметриями нелинейных систем". Здесь следует отметить, что если в используемом конкретном наборе квазиинвариантов присутствует квазиинвариант, которьй являлся знаменателем некоторой дроби (производной), то данньй конкретный набор квазиинвариантов обращает в нуль и числитель этой дроби. Никаких особенностей не возникает.

ЗАмечАниЕ 2.1. Заметим, что уравнение Гамильтона-Якоби для функции $H(x, t)$ (1.32) в теореме 1.1 и является квазиинвариантом, так как вьведено по предлагаемой схеме.

ПримеР 5. В качестве примера приведем утверждение 
УТВЕРЖДЕНИЕ 2.1. Пусть в уравнении (1.3) $\varphi_{1}=1, \varphi_{3}=1, k_{0}=1 ;$ выберем из списка квазиинвариантов

$$
\begin{gathered}
Q=0, \quad Q_{1}=0, \quad Z_{2}=\frac{1}{4} Z_{1}, \\
2\left(-1+\sigma Z_{1}(x, t)\right) Z_{1}(x, t) H_{x}+\left(-1+\sigma Z_{1}(x, t)\right) Z_{1_{x}}=0,
\end{gathered}
$$

где $\sigma= \pm 1$. Тогда исправленный анзаи, решения уравнения (1.3) имеет вид

$$
u(x, t)=\frac{Q_{2}(x, t)}{Z_{1}(x, t)\left(C_{1}+Z_{1}(x, t)\right)+C_{2} \sqrt{\left|Z_{1}(x, t)\right|} \sqrt{\left|1+Z_{1}(x, t)\right|}} .
$$

ДоКАЗАТЕЛЬСТВО проводится подстановкой анзаца решения второй моды в уравнение (1.3). Вычислим квазиинварианты. Приравнивая выбранные из них к нулю, получим (2.3). Подставляем этот анзац в уравнение (1.3) и групшируем слагаемые при $\sqrt{\left|1+Z_{1}(x, t)\right|}, \sqrt{\left|Z_{1}(x, t)\right|}$. Получим систему двух уравнений на две функции $Q_{2}(x, t)$, $Z_{1}(x, t)$. Таким образом, анзац метода Хироты трансформировался в анзац $(2.3)$. Этот анзац обладает свойством самоподобного усложнения. В его знаменатель можно набирать следующие слагаемые, как усложняющуюся композицию уже имеющихся слагаемых. Если перейти в полученной системе к переменной $a x+b t$, получится система ОДУ. График решения задачи Коши для нее приведен на рис. 4 (верхняя кривая, $\left.C_{1}=1, C_{2}=1, a=\sqrt{2}, b=-3 / 2\right)$. Если дополнительно предположить, что $Q_{2}(x, t)=$ $f\left(Z_{1}(x, t)\right)$, то в переменных простой волны $\tau=a x+b t, \xi=Z_{1}(x, t)$ два уравнения системы вырождаются в одинаковое ОДУ первого порядка:

$$
4 f^{\prime}(\xi) f(\xi) \xi(1+\xi)-(5+8 \xi) f(\xi)^{2}+\xi^{2}+\xi^{3}=0
$$

Интегрируя последнее уравнение, получим решение уравнения (1.3)

$$
u(x, t)=\left.\frac{\sqrt{|1+z(\tau)|} \sqrt{2+4 z(\tau)-4 \sqrt{|z(\tau)(1+z(\tau))|}}}{\sqrt{2}(1+z(\tau)+\sqrt{|z(\tau)(1+z(\tau))|})}\right|_{\tau=a x+b t},
$$

где $z(\tau)=\left.Z_{1}(x, t)\right|_{\tau=a x+b t}$. Для этой функции имеем одно ОДУ второго порядка (нижняя кривая на рис. 4 изображает решение задачи Коши при тех же значениях констант, что и верхняя кривая). Такие решения могут быть полезными для теорем сравнения [29]. Построенные решения, если использовать их как начальные условия задачи Коши для уравнения в частных производных (1.3), эволюционируют к тождественной единице.

3. “Системы уравнений и модификация метода Хироты”. Рассмотрим систему уравнений (Кавахара, Танака). Полная библиография приведена в работе [14], где изучались ее точные решения

$$
\begin{aligned}
& u_{t}-u_{x x}-u+u v^{n}+u^{m+1}=0 \\
& v_{t}-v_{x x}+B v u^{2}=0 .
\end{aligned}
$$




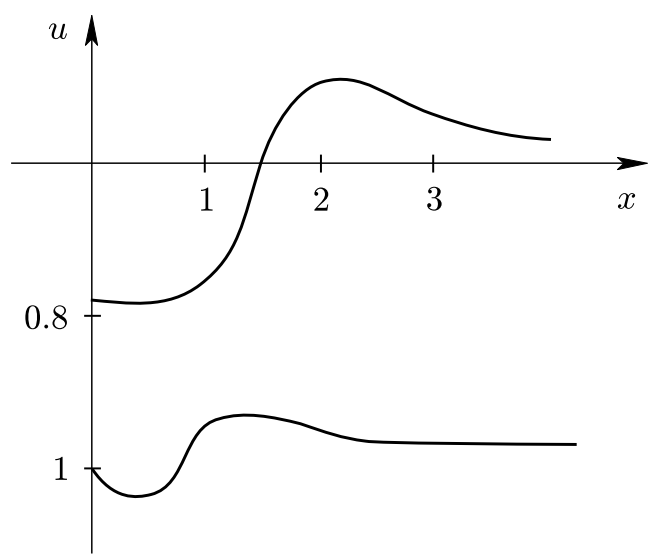

Рис. 4

Рассмотрим функции (два решения системы) $M_{0}, M_{1}, Q_{0}, Q_{1} \in C\left[\mathbb{R}^{1} \times \mathbb{R}_{+}^{1}\right]$ и поставим им в соответствие вектор $u(x, t), v(x, t)$ (операция $\diamond)$ :

$$
u(x, t)=\frac{M_{0}+M_{1} \exp (H(x, t))}{1+\exp (H(x, t))}, \quad v(x, t)=\frac{Q_{0}+Q_{1} \exp (H(x, t))}{1+\exp (H(x, t))},
$$

где функция $H(x, t)$ имеет вид

$$
H(x, t)=C_{1}+\frac{1}{\sqrt{2}} \int \sqrt{M_{0}^{2}-2 M_{0} M_{1}+M_{1}^{2}+\left(Q_{0}-Q_{1}\right)^{2}} d x
$$

$C_{1}$ - константа.

УТВЕРЖДЕНИЕ 3.1. Решение системы (3.1) при $n=2, m=2, B=2$, построенное по паре решений

$$
\begin{gathered}
M_{0}=\frac{1-\exp \left(C_{0}+x-t\right)}{1+\exp (x-t)+(x-1+2 t) \exp \left(C_{0}+x-t\right)}, \quad M_{1}=\frac{1}{1-2 t-x}, \\
Q_{0}=\frac{1+(2 t+x) \exp C_{0}}{1+\exp (-x+t)+(x-1+2 t) \exp C_{0}}, \quad Q_{1}=\frac{-2 t-x}{1-2 t-x}, \\
H[x, t]=C_{1}+x+\ln (x+2 t-1)-\ln \left(\exp t+\exp x+(x-1+2 t) \exp \left(C_{0}+x\right)\right),
\end{gathered}
$$

имеет вид

$$
\begin{aligned}
& u=\frac{1-\exp \left(C_{0}+x-t\right)-\exp \left(C_{1}+x-t\right)}{1+\exp (x-t)+(x-1+2 t)\left(\exp \left(C_{1}+x-t\right)+\exp \left(C_{0}+x-t\right)\right)}, \\
& v=\frac{1+(x+2 t) \exp \left(C_{0}\right)+(x+2 t) \exp \left(C_{1}\right)}{1+\exp (-x+t)+(x-1+2 t)\left(\exp \left(C_{1}\right)+\exp \left(C_{0}\right)\right)}
\end{aligned}
$$

Здесь по точным однофазным решениям с помощью (3.2) строятся сначала решения (3.4), а затем строятся решения (3.5). 
УТВеРЖДЕНИЕ 3.2. Решение системы (3.1) nри $n=1, m=2$, построенное по паре решений

$$
\begin{aligned}
M_{0}= & \frac{1}{1+\exp (-t / 2 \pm x / \sqrt{2})}, \\
M_{1}= & \frac{1+\exp C_{1}-\exp \left(C_{1} \pm \sqrt{2} x\right)}{1+\exp C_{1}+\exp (-t / 2 \pm x / \sqrt{2})+\exp \left(C_{1} \pm \sqrt{2} x\right)} \\
Q_{0}= & \frac{\exp (-t / 2 \pm x / \sqrt{2})}{1+\exp (-t / 2 \pm x / \sqrt{2})}, \\
Q_{1}= & \frac{1}{1+\left(1+\exp C_{1}\right) \exp (-t / 2 \mp x / \sqrt{2})+\exp \left(C_{1}\right) \exp (t / 2 \pm x / \sqrt{2})}, \\
H[x, t]= & C_{2}-\ln \left(1+\exp \left(-\frac{t}{2} \pm \frac{x}{\sqrt{2}}\right)\right) \\
& \quad+\ln \left(1+\exp \left(C_{1}\right)+\exp \left(-\frac{t}{2} \pm \frac{x}{\sqrt{2}}\right)+\exp \left(C_{1} \pm \sqrt{2} x\right)\right)
\end{aligned}
$$

имеет вид

$$
\begin{aligned}
& u=\frac{1+\exp C_{2}+\exp \left(C_{1}+C_{2}\right)-\exp \left(C_{1}+C_{2} \pm \sqrt{2} x\right)}{1+\exp C_{2}+\exp \left(C_{1}+C_{2}\right)+\left(1+\exp C_{2}\right)(\exp (-t / 2 \pm x / \sqrt{2}))}, \\
& v=\frac{\left(1+\exp C_{2}\right) \exp (-t / 2 \pm x / \sqrt{2})}{\left(1+\exp C_{2}\right) \exp (-t / 2 \pm x / \sqrt{2})+\exp \left(C_{1}+C_{2}\right)(1+\exp ( \pm \sqrt{2} x))}
\end{aligned}
$$

В заключение отметим, что такой подход переносится на многомерньй случай.

Автор благодарен В. Г. Данилову и С. Ю. Доброхотову за постоянное внимание к работе и полезные обсуждения, а также В. П. Маслову, А. Д. Полянину, Г. А. Омельянову, А.И. Шафаревичу и Н. Эйлеру за конструктивные советы.

\section{СПИСОК ЦИТИРОВАННОЙ ЛИТЕРАТУРЫ}

[1] Volosov K. A. Invariant properties of the ansatz of the Hirota method for quasilinear parabolic equations // International conference "Differential Equations and Related Topics". XX Joint Session of Petrovskii Seminar and Moscow Mathematical Society. M., 2001. P. 433.

[2] Volosov K.A. Tools for mathematical modeling // The Third International Conference. Saint-Petersburg, 2001.

[3] Danilov V. G., Maslov V.P., Volosov K. A. Mathematical Modelling of Heat and Mass Transfer Processes. Dordrecht-Boston-London: Kluwer Acad. Publ., 1995.

[4] Данилов В. Г., Субочев П. Ю. Кинковые решения в уравнении КПП-Фишера // Матем. заметки. 1991. Т. 50. № 3. С. 152-154.

[5] Hirota R. Exact solution of the Korteweg-de Vries equations for the multiple collisions of solitons // J. Phys. Soc. Japan. 1972. V. 33. P. 1459.

[6] Новиков С. П. (ред. ) Солитоны. М.: Мир, 1983.

[7] Новиков С.П. (ред. ) Волны в активных и нелинейных средах в приложении к электронике. М.: Мир, 1977.

[8] Goldstone J., Jaskiw R. Quantization of nonlinear waves // Phys. Rev. D. 1975. V. 11. P. 1486.

[9] Dashen R.F., Hasslacher B., Neveu A. Nonperturbative methods and extended-hadron models in field theory I-III // Phys. Rev. D. 1974. V. 10. P. 4114-4129, 4130-4137, $4138-4142$. 
[10] Ablowitz M. J., Zeppeteller P. Explicit solutions of Fisher's equations for a special wave speed // Zeppeteller Bul. Math. Biol. 1979. V. 41. P. 835-840.

[11] Данилов В.Г., Омельянов Г.А., Радкевич Е.В. Обоснование асимптотического решения для системы фазового поля и модифицированная задача Стефана // Матем. сб. 1996. T. 186. №12. C. $63-80$.

[12] Ablowitz M. J., Segur H. Solution for Inverse Scattering Transform. Philadelphia: SIAM, 1981.

[13] Маслов В. П., Данилов В. Г., Волосов К. А. Математическое моделирование процессов тепломассопереноса (эволюция диссипативных структур). С добавлением Н. А. Колобова. М.: Наука, 1987.

[14] Волосов К. А., Данилов В. Г., Логинов А. М. Точные автомодельные и двухфазные решения систем полулинейных параболических уравнений // ТМФ. 1994. Т. 101. № 2 . C. $189-199$.

[15] Fisher R. A. The wave of advance of advantageous Genes // Ann. of Eugenics. 1937. V. 7. P. 355-369.

[16] Данилов В. Г., Омельянов Г. А., Радкевич Е. В.Асимптотика решения системы фазового поля и модифицированная задача Стефана // Дифференц. уравнения. 1995. Т. 31. № 3 . C. $483-491$.

[17] Данилов В. Г., Омельянов Г. А., Радкевич Е. В. О регуляризации данных модифицированной задачи Стефана // Матем. заметки. 1995. Т. 57. № 4. С. 793-795.

[18] Маслов В. П., Омельянов Г. А. Асимптотические солитонообразные решения уравнений с малой дисперсией // УМН. 1981. Т. 36. № 3. С. 63-126.

[19] Маслов В. П., Омельянов Г. А. Об условиях типа Гюгонио для бесконечно узких решений уравнения простых волн // Сиб. матем. ж. 1983. Т. 24. № 5. С. 172-181.

[20] Волосов К.А., Данилов В.Г., Колобов Н. А., Маслов В.П. Локализованные уединенные волны // Докл. АН СССР. 1986. Т. 287. №6. С. 535-538.

[21] Зайцев В.Ф., Полянин А. Д. Справочник. Обыкновенные дифференциальне уравнения. М.: Физматлит, 2001.

[22] Белотелов Н. В., Лобанов А. И. Популяционные модели нелинейной диффузии // Матем. модел. 1997. Т. 9. № 12. С. 43-56.

[23] Лобанов А. И., Старожилова Т. К. Качественное исследование начального этапа формирования неравновесных структур в модели типа "реакция-диффузия" // Матем. модел. 1997. T. 9. № 12. C. 23-26.

[24] Мельникова Е. В. Нелинейная динамика распространения эпидемий // Изв. вузов. Прикл. нелин. динам. 1998. Т. 6. № 2. С. 110-116.

[25] Кожонов А. И.Краевая задача для одного класса параболических уравнений, возникающая при описании процесса опреснения // Сб. науч. трудов. Сиб. отд. АН СССР. Ин-т гидродинамики. 1978. №36. С. 38-46.

[26] Волосов К. А. Инвариантные свойства анзаца метода R. Hirota. Новые информационне технологии. Материалы четвертого семинара. М.: МГИЭМ(ТУ), 2001.

[27] Пухначев В.В. Преобразования эквивалентности и скрытая симметрия эволюционных уравнений // Докл. АН СССР. 1987. Т. 294. №3. С. 535-538.

[28] Галактионов В.А., Посашков С. А. Точные решения и инвариантные пространства для нелинейных уравнений градиентной диффузии // ЖВМиМФ. 1994. Т. 34. С. 374-383.

[29] Gilding B.H., Kersner R. The characterization of reaction-convection-diffusion processes by travelling waves // J. Differential Equations. 1996. V. 124. №1. P. 27-29. 